\title{
The calcium binding properties and structure prediction of the Hax-1 protein
}

\author{
Anna Balcerak, Sebastian Rowinski, Lukasz M. Szafron and Ewa A. Grzybowska \\ Cancer Center Institute, Warsaw, Poland
}

Hax-1 is a protein involved in regulation of different cellular processes, but its properties and exact mechanisms of action remain unknown. In this work, using purified, recombinant Hax-1 and by applying an in vitro autoradiography assay we have shown that this protein binds $\mathrm{Ca}^{2+}$. Additionally, we performed structure prediction analysis which shows that Hax-1 displays definitive structural features, such as two $\alpha$-helices, short $\beta$-strands and four disordered segments.

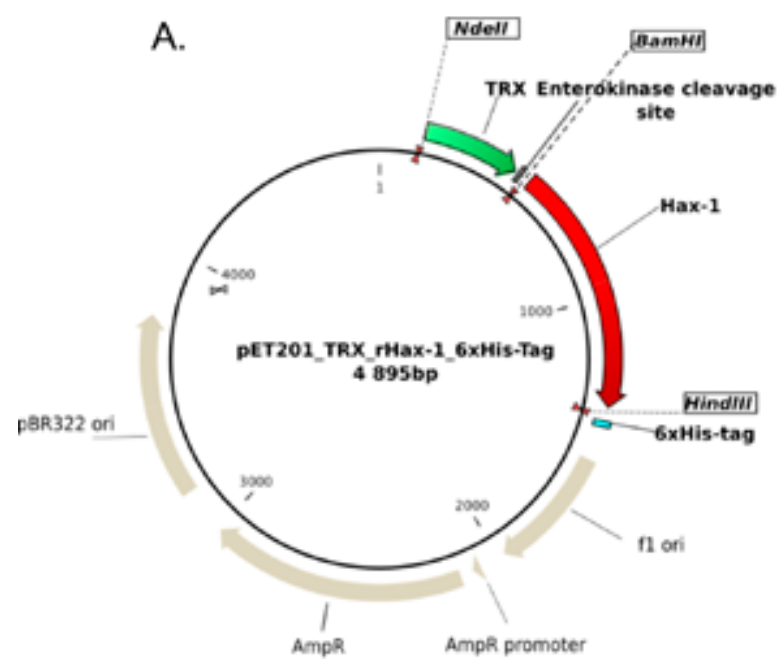

Key words: Hax-1, tags removal, calcium binding, 3D protein model Received: 21 February, 2017; revised: 19 April, 2017; accepted: 07 June, 2017; available on-line: 01 September, 2017

e-mail: ewag@coi.waw.pl

Abbreviations: PONDR, predictor of natural disordered regions; IDPs, intrinsically disordered proteins

Supplementary Figure 1. Generation of the pET201_6xHis_TRX_Hax-1 expression vector. Restriction sites used in cloning and the relevant features of the two vectors are marked.

(A) A map of the initial pET201_TRX_Hax-1_6xHis vector used in cloning. (B) A map of the modified pET201_6xHis_TRX_Hax-1 expression vector. The vector was re-designed to encode the His-tag at the $\mathrm{N}$-terminus, in frame with thioredoxin, and to destroy the $\mathrm{C}$-terminal His-tag by the addition of two stop codons. 


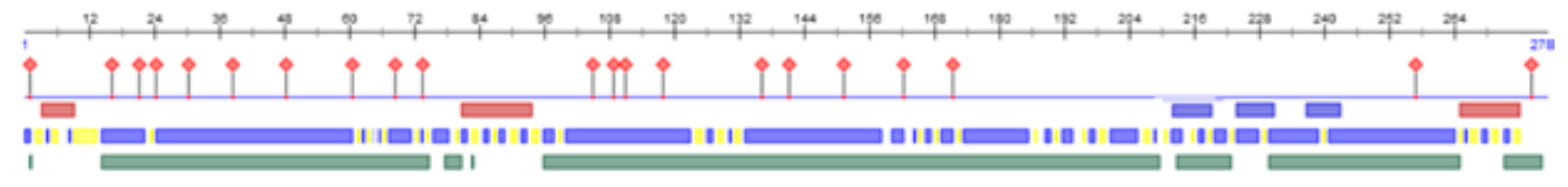

Legend.

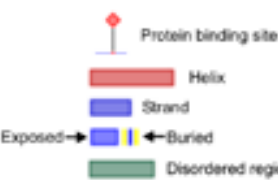

Supplementary Figure 2. Rat Hax-1 secondary structure prediction (PredictProtein 2013).

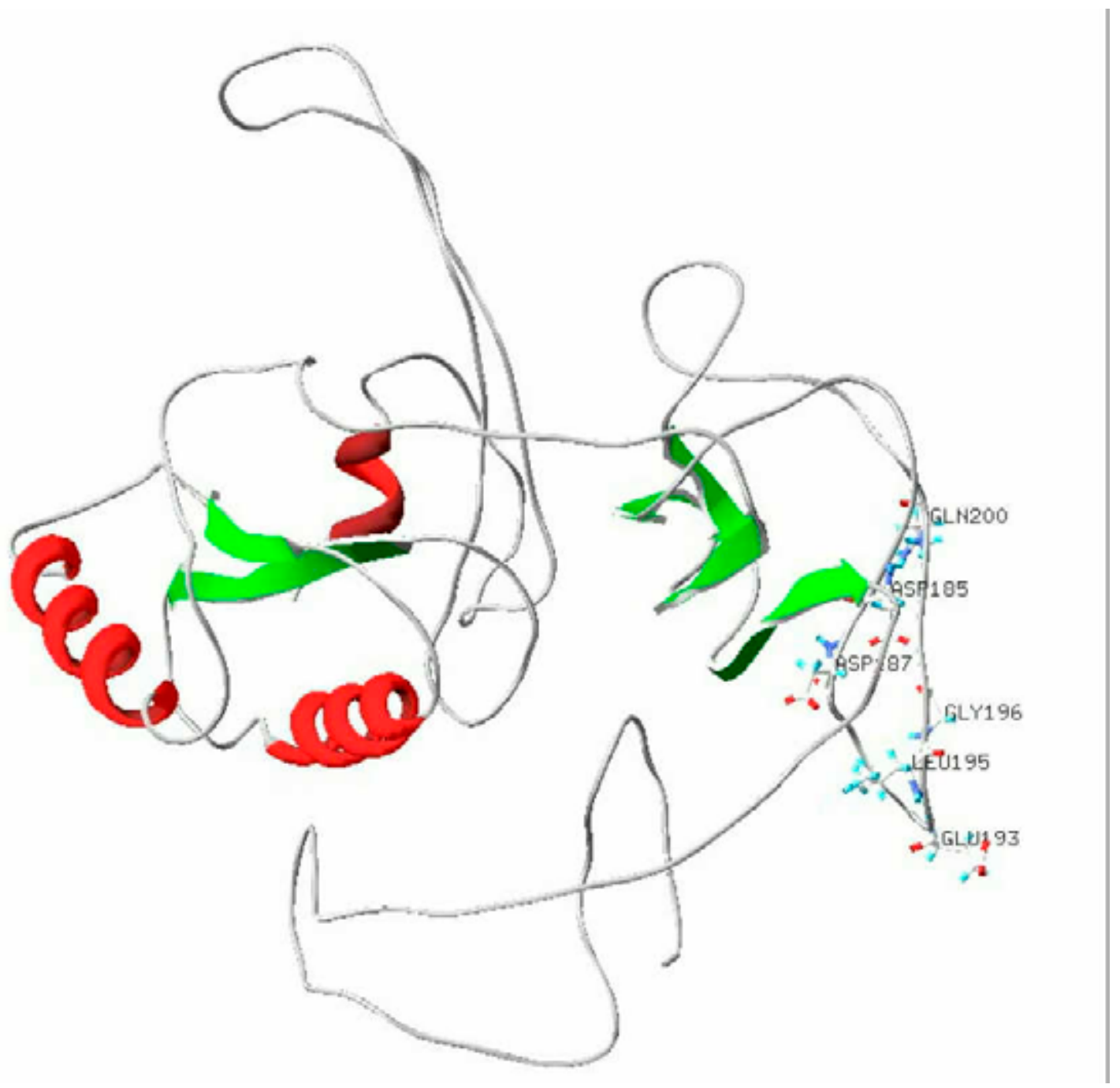

Video 1. An animation of the rat Hax-1 protein model, depicted in Fig. 4.

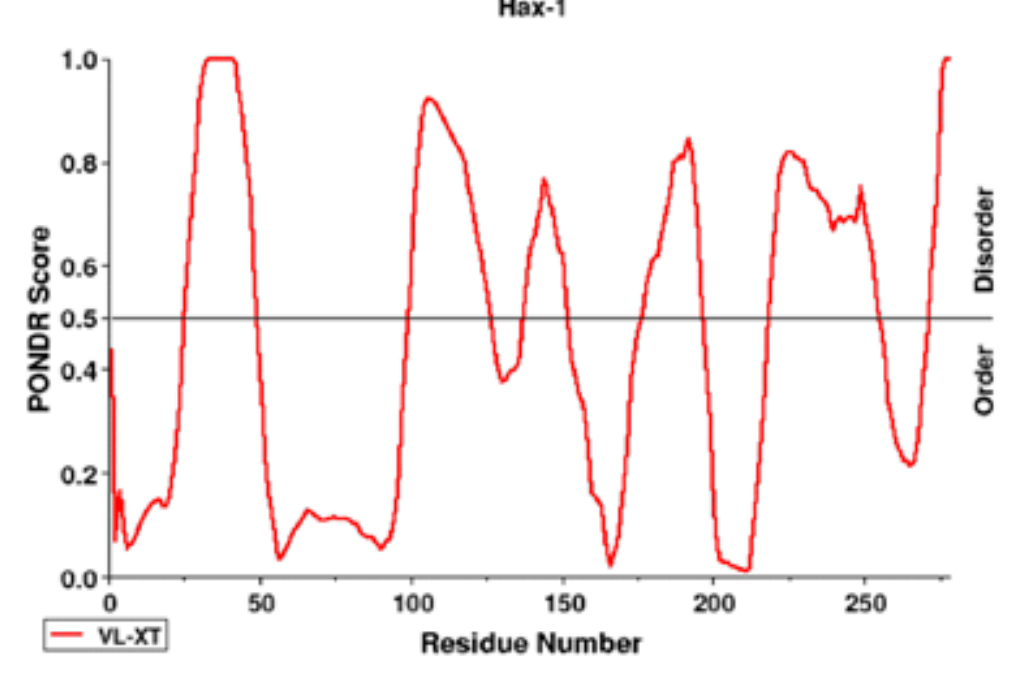

PONDR Protein Disorder Predictor

Developed by P. Romero, X. Li, A.K. Dunker,Z. Obradovic, E. Garner.

VL3 Predicto

Developed by P. Radivojac and A.K. Dunker

Developed by P. Radivojac

SLL2 Predictor

Developped by K. Peng and Z. Obradovic

Prestist

Predicted residues: 278 Number Disordered Regions: 6

Overall percent disordered: 46.04 Average Prediltion Score: 0.4567

Predicted disorder segment [266-[49]/Average Strength $=0.8749$

Predicted disorder segment [100]-[126]Average Strength $=0.7811$

Predicted disorder segment (135). (151) Average Strength $=0.6638$

Predicted disorder segment [2191. [254]Average Strength $=0.7189$

Predicted disorder segment [272]-[278]Average Strength $=0.8322$

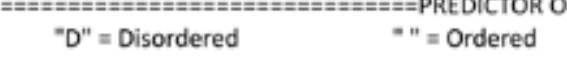

1 MSVFDLFRGF FGFPGPRSHR DPFFGGMTRD DDDDEDDEEE EDSGAWGRES

VLXT DDDDD DODDDDODDD DDDDDODDO

S1 YAFDGFHPTE EFGFFFSPRG GMRFHGNFGF DDLVRDFNSI FSEMGAWTLP

101 SHSPELPGPE SETPGVRLRE GQTLROSMLK YPOSHQPRIF EGVLESHAKP 0
0

151 ESSKPAPDWG SQGPFHRLDD TWPVSPHSRA REDKDLDSQV SQEGLGPLLO VXT D DOD D

201 PQPKSYFKSI SVTKITKPDG TVEEHRTVVD SEGRRETTVT HQEAHDSSRS VLXT DD DODDDDDDDD DDDDDDDDOD DDDDDDDDDD

251 DPDPPRSSAL DDPFSIDDH LGRWFPS VLXT DDDD DDDDDDD

Supplementary Figure 3. Rat Hax-1 analyzed by PONDR. 